\title{
Effect of Remote Distractors on Saccade Programming: Evidence for an Extended Fixation Zone
}

\author{
ROBIN WALKER, ${ }^{1}$ HEINER DEUBEL, ${ }^{2}$ WERNER X. SCHNEIDER, ${ }^{2}$ AND JOHN M. FINDLAY ${ }^{1}$ \\ ${ }^{1}$ Department of Psychology, University of Durham, Durham, DH1 3LE United Kingdom; and ${ }^{2}$ Institut für Psychologie, \\ Allgemeine und Experimentelle Psychologie, D-80802 Munich, Germany
}

\begin{abstract}
Walker, Robin, Heiner Deubel, Werner X. Schneider, and John M. Findlay. Effect of remote distractors on saccade programming: evidence for an extended fixation zone. J. Neurophysiol. 78: 1108$1119,1997$. In a series of experiments, we examined the increase in saccade latency that is observed consistently when distractor stimuli are presented simultaneously with the saccade target at various nontarget locations. In the first experiment, targets and distractors were presented on the horizontal axis. We found that saccade latency was increased when distractors appeared at fixation and in the contralateral nontarget hemifield (at eccentricities $\leq 10^{\circ}$ ). In contrast, latency was unaffected by distractors presented along the ipsilateral target axis, but amplitude was increased as saccades tended to land at intermediate locations between the two stimuli (global effect). The effect of presenting distractors at various two-dimensional locations in both the target and nontarget hemifields then was examined, and the maximum latency increase again was observed when distractors appeared at fixation. Distractors presented on any of the eight principal axes in either hemifield, other than on the horizontal target axis, also increased latency. The relationship between the effects of distractors on latency and amplitude was reciprocal. Within $\sim 20^{\circ}$ of the target axis itself, distractors affected saccade amplitude but not latency. In contrast, distractors presented outside this "window" increased saccade latency without affecting amplitude. A systematic quantitative relationship was revealed between the increase in latency and the ratio between target and distractor eccentricities. The latency increase was largest with small values of the ratio and reached a peak with distractors at the fixation location. The finding that the increase observed for more eccentric distractor locations fitted the same function as that at fixation shows that inhibitory effects operate over large areas of the visual field. The increase in latency under distractor conditions is interpreted in light of recent neurophysiological findings of inhibitory processes operating in the rostral region of the superior colliculus. Our results suggest that these inhibitory processes are not restricted to the central foveal region alone but operate over wider regions of the visual field.
\end{abstract}

\section{INTRODUCTION}

Saccadic eye movements are fast movements of the eyes that are made to bring novel objects and stimuli onto the fovea for detailed analysis. Experimental studies of saccadic eye movements to visual targets have proved to be a highly successful method of studying the neural basis of a simple behavioral response. It has been shown that subtle manipulations of the central fixation stimulus (Fischer and Breitmeyer 1987; Reuter-Lorenz et al. 1991, 1995; Saslow 1967), stimulus eccentricity (Kalesnykas and Hallett 1994; Weber et al. 1992; Wyman and Steinman 1973), and number of stimuli (Deubel et al. 1984; Findlay 1982; Lévy-Schoen and BlancGarin 1974) can produce differential effects on the temporal and spatial parameters of saccades. The close correspondence between the underlying neurophysiology of the saccadic system and the behavioral data has resulted in explanations of the behavioral data in terms of specific neural activity (Findlay 1987; Fischer 1987; Munoz and Wurtz 1995a,b; Reuter-Lorenz et al. 1995).

The gap paradigm is one experimental manipulation known to influence the temporal aspects of saccade programming. In the gap situation, the offset of fixation at some interval (gap) before the onset of the peripheral target results in a systematic reduction in latency (Saslow 1967) and also may increase the numbers of short latency "express saccades" observed (Fischer and Ramsperger 1984). It has been suggested that the neurophysiological substrate of the gap effect may be found in the activity of cells in the rostral pole of the superior colliculus. Cells in this region share some of the characteristics of the brain stem "pause" cells in that they are active during fixation but their activity diminishes before a saccadic eye movement (Munoz and Wurtz 1993a). Dorris and Munoz (1995) have made the specific suggestion that visual stimulation can affect this region and lead to the phenomenon of the gap facilitation effect.

Another manipulation known to affect aspects of saccade programming is the situation in which a distractor is presented at nontarget locations. Studies using distractors have been show to influence both the temporal (Lévy-Schoen 1969) and spatial (Deubel et al. 1984; Findlay 1982) parameters of saccades. One revealing experimental situation examines the saccades produced when two targets are presented simultaneously in the visual field. If the two targets are remote, then an accurate saccade is made to one, but the latency for the saccade is prolonged over that when only a single target is presented. Lévy-Schoen (1969) first showed that saccade latency is increased (by $40 \mathrm{~ms}$ in the conditions of her study) when a distractor appears simultaneously in the mirror symmetric position of the contralateral hemifield. If, on the other hand, two targets are presented in neighboring positions, no latency increase is found but saccade amplitudes are affected. Under conditions in which pairs of targets are presented along the target axis saccades tend to be directed to the "center of gravity" of the target configuration and an increase in amplitude termed the "global effect' ' is observed (Deubel et al. 1984; Findlay 1982). These differential effects are consistent with the view that separate parallel processes are involved in programming the decision to initiate a saccade (WHEN) and the computation of the spatial parameters (WHERE) of the saccade (Becker and 
Jürgens 1979; Findlay 1983). Remote distractors affect the WHEN process, whereas neighboring distractors affect the WHERE process.

Although studies of the gap paradigm have been highly influential in the development of models of the saccadic system (Fischer 1987; Munoz and Wurtz 1995a,b; ReuterLorenz et al. 1995), few studies have examined the effects of presenting remote distractors on saccade generation. The latency increase observed when distractors appear bilaterally at mirror symmetric positions has been studied in primates with brain lesions (Lynch and McLaren 1989) and in human patients with cortical damage (Rafal et al. 1990; Walker et al. 1991, 1996), but few systematic examinations of the remote distractor effect have been performed with "normal", human subjects.

One notable exception is the study by Weber and Fischer (1994), who examined the effects of distractors on the generation of short latency express saccades. In their first experiment, subjects made saccades to a target that appeared randomly to the left or right of fixation. In half the trials, a distractor (a vertical strip of 3 bars) appeared at the mirror symmetric location in the opposite hemifield. The latency of saccades was increased and express saccades were suppressed in trials in which a distractor appeared. Under these conditions it is unclear, however, if the latency increase on distractor trials is due to the appearance of the distractor or to the additional discrimination process required to select the target on bilateral trials. In subsequent experiments, a single saccade direction was maintained and an increase in latency was again observed on distractor trials, showing that the effect is not due to the discrimination process. In further studies, it was found that both the size and eccentricity of the contralateral distractor influenced the magnitude of the inhibitory effect on saccade latency. Small distractors $\left(0.1 \times 0.1^{\circ}\right)$ had less of an inhibitory effect than larger distractors $\left(0.4 \times 0.4^{\circ}\right)$, and distractors presented at $12^{\circ}$ had less inhibitory effect than did distractors at $4^{\circ}$. The effects of presenting a distractor (a vertical strip of 23 bars) at various eccentricities in the ipsilateral (target) hemifield also was investigated. It was found that ipsilateral distractors reduced the numbers of express saccades when presented close to the fixation region or dead zone (Weber et al. 1992). As the size of the distractor stimulus was varied across studies, it is not possible to directly compare the inhibitory effects observed with ipsilateral and contralateral distractors in the Weber and Fischer study.

Walker et al. (1995) also examined the effects of presenting distractors in the contralateral hemifield on human saccade latency under conditions in which a single saccade direction was maintained. The results of the first experiment were consistent with the findings of Weber and Fischer (1994) and showed that distractors presented bilaterally and simultaneously in the contralateral hemifield can increase saccade latency by some 20-30 ms. In a further experiment, the temporal aspects of the remote distractor effect were investigated by manipulating the timing of the distractor onset relative to that of the target. The maximum latency increase was observed when the distractor appeared simultaneously with the target onset. Latency also was increased if the distractor preceded the target by an interval of $<100 \mathrm{~ms}$. When the contralateral distractor appeared at intervals of
$>100-250 \mathrm{~ms}$ before the onset of the saccade target, a systematic latency decrease was observed, possibly attributable to warning signal effects (Ross and Ross 1980, 1981).

It appears plausible to suggest that detailed examinations of the remote distractor paradigm could be as influential as studies of the gap effect in guiding our understanding of the saccadic system. Furthermore, the increase in latency observed in the remote distractor condition may be linked to inhibitory processes known to operate in structures involved in saccade generation such as the superior colliculus (Munoz and Wurtz 1992; Munoz et al. 1996). Previous studies of the remote distractor effect have, as noted above, been restricted to observing the effects of presenting distractors at the "mirror image" location in the opposite visual field to the target. The sole exception is Weber and Fischer's 1994 study, which was concentrated on the production of express saccades, and, as already mentioned, the inhibitory effects of ipsilateral and contralateral distractors cannot be directly compared as the size of the distractor stimulus was varied. In the work reported here, we have extended our understanding in the following series of experiments by presenting distractors at various locations in both visual fields and performing a systematic examination of the effects on saccade latency and amplitude. We report the results of three experiments in which a detailed examination of the spatial location of the remote distractor on saccade latency was performed while the size of the distractor was held constant. A reciprocal relationship between latency and amplitude effects was observed; distractors presented within $\pm 20^{\circ}$ of the target axis modulated amplitude but not latency, and distractors presented at any location outside this window increased latency but did not influence amplitude. A systematic relationship was observed between the ratio of distractor and target eccentricity and the magnitude of the latency increase; the greatest increase in latency was observed when the distractor was presented at fixation. This suggests that the remote distractor effect may involve similar inhibitory mechanisms to those of the gap effect and thus also may involve processes of competitive inhibition from the rostral pole region of the superior colliculus (Munoz and Wurtz 1993a,b; Munoz et al. 1996).

\section{METHODS}

\section{Subjects}

Six subjects participated in each experiment and one additional subject participated in experiment 2 . All were laboratory workers from the Max-Planck institute (age 21-53 yr).

\section{Apparatus}

Subjects viewed a fast 21 -in color monitor with a frame rate of $100 \mathrm{~Hz}$ at a distance of $80 \mathrm{~cm}$. The video signals were generated by a programmable graphics board controlled by a PC via a TIGA (Texas Instruments graphics adapter) interface. Eye movements were recorded using a Generation 5.5 Dual-Purkinje eye tracker (Crane and Steele 1985), which provides accurate measurements of both saccade latency and amplitude. The analogue eye movement signal was sampled at a rate of $400 \mathrm{~Hz}$ and stored onto disk for later off-line analysis. Head movements were restricted by use of a biteboard and forehead rest.

A calibration routine was performed before each testing session 
in which subjects were required to sequentially fixate 10 positions arranged on a circular array of $6^{\circ}$ radius. An automatic saccade analysis program was used to analyze the data off-line. Saccade starting and endpoints were calculated using a velocity criterion of $30 \%$ s. As eye-lens deviations result in an artifactual overshoot of final eye position with Purkinje tracking techniques (Deubel and Bridgeman 1995), the program searched for a location after the overshoot to calculate saccade amplitude in relation to the initial calibration. Saccades with latency $<80 \mathrm{~ms}$ were excluded on the grounds that they were probably anticipatory (Fischer et al. 1993; Wenban-Smith and Findlay 1991), and saccades with latency $>450 \mathrm{~ms}$ were excluded as not being visually triggered.

\section{Stimulus presentation}

The following stimulus sequence was common to each experiment. At the start of each trial, a small cross (width $0.19^{\circ}$ ) appeared in the center of the screen for a variable period of between 500 and $1,000 \mathrm{~ms}$. The target stimulus was a diagonal cross (length $0.19^{\circ}$, line width 2 pixels ) presented on a horizontal axis level with fixation. Targets appeared simultaneously with the offset of the fixation cross (0 Gap). A single saccade direction (left or right) was maintained throughout a block of trials, and each subject completed a block of trials for each direction (counterbalanced). Each block contained 10 trials per condition (producing 20 data points per condition if data from 2 blocks are collapsed).

The stimulus locations used in each experiment are displayed schematically in Fig. 1. In experiments $1 a, 2$, and 3, targets appeared at eccentricities of 4 and $8^{\circ}$, a range where a stable latency function is observed (Kalesnykas and Hallett 1994). In experiment $1 b$, targets were presented at eccentricities closer to the fovea $\left(0.5-4^{\circ}\right)$ where an increase in saccade latency is observed (Kalesnykas and Hallett 1994; Weber et al. 1992). In the majority of trials, a single distractor ( $\bigcirc$ diameter $0.53^{\circ}, 2$ pixels thick) ap-
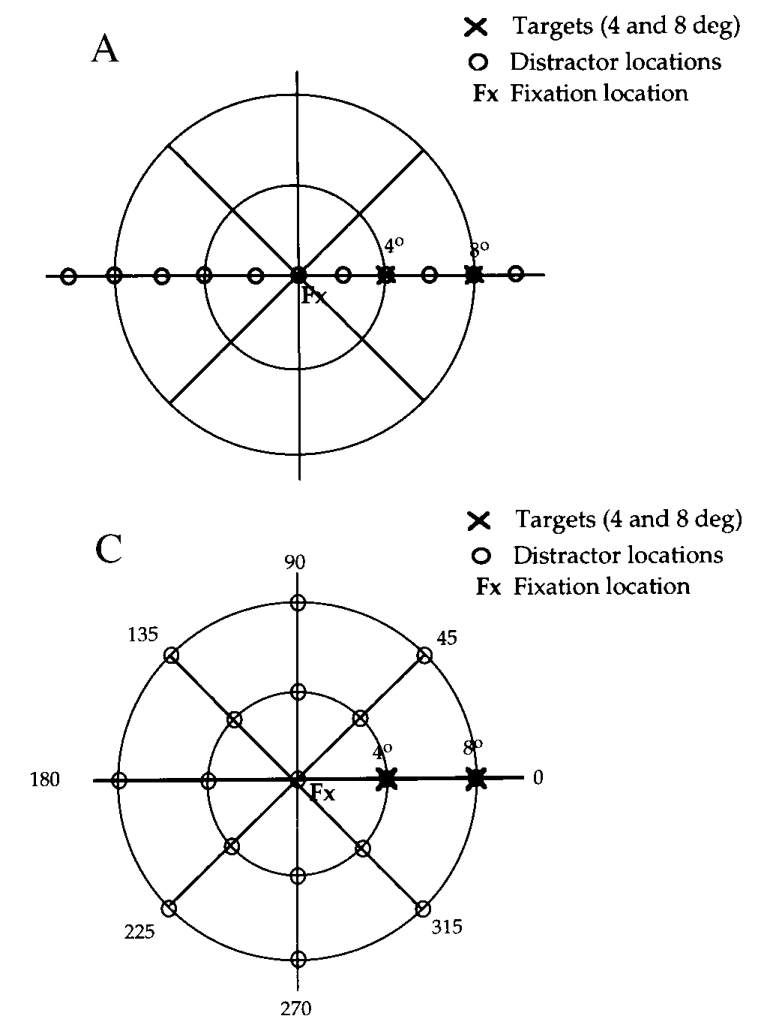

peared simultaneously with the onset of the saccade target. Subjects were informed in advance of the direction in which the target would appear and were instructed to move their eyes as quickly as possible to the targets while ignoring the distractors. The spatial location of the distractors and eccentricity of the target was the main manipulation for each experiment. The stimulus sequence for each saccade paradigm is described in the following section.

\section{Experiment 1a}

In experiment $1 a$, targets appeared at eccentricities of 4 and $8^{\circ}$ along a horizontal axis. On some trials a single target appeared, while on others distractors were presented at various eccentricities $\left(-10,-8,-6,-4,-2,0,2,4,6,8,10^{\circ}\right)$ in both hemifields (see Fig. $1 A)$. Where: 0 indicates a distractor presented at the central fixation location, - ve indicates distractors in the opposite (contralateral) hemifield, and + ve indicates distractors in the same (ipsilateral) hemifield as the target. Subjects completed two blocks (left and right saccades) of 240 trials.

\section{Experiment $1 b$}

In experiment $1 b$, targets were presented along a horizontal axis at various eccentricities $\left(0.5,1,2\right.$, and $\left.4^{\circ}\right)$ close to the fovea. In some trials, a single target appeared, whereas in others, a distractor was presented at fixation $(0)$, at $-8,-4,-2,-1$, or $-0.5^{\circ}$ in the contralateral hemifield, or $+4^{\circ}$ in the ipsilateral hemifield (see Fig. $1 B)$. Subjects completed two blocks (left and right saccades) of 320 trials.

\section{Experiment 2}

In experiment 2 , targets appeared at 4 and $8^{\circ}$ along a horizontal axis. In some trials a single target appeared, whereas in others, a

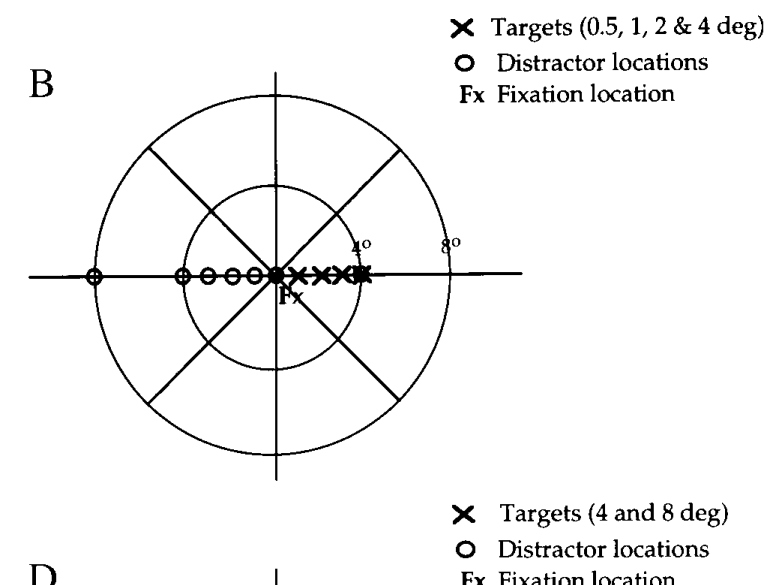

$\mathrm{D}$

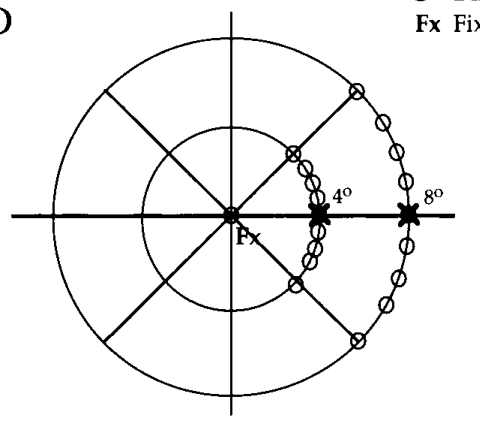

FIG. 1. Schematic representation of stimuli positions used in each experiment. A: experiment $1 a$; B: experiment $1 b$; $C$ : experiment $2 ; D$ : experiment 3 . 
distractor appeared simultaneously at one of the 17 possible twodimensional (2-D) locations. Distractors were presented at fixation (fx) or at 4 and $8^{\circ}$ eccentricities at various 2-D locations on each of the eight principal axes $(0,+45,+90,+135,180,-45,-90$, and $-135^{\circ}$ ), in both hemifields (see Fig. $1 C$ ). Subjects completed two blocks (left and right saccades) of 360 trials.

\section{Experiment 3}

In experiment 3 , targets again appeared at 4 and $8^{\circ}$ along a horizontal axis. Distractors appeared at fixation (fx) or at eccentricities of 4 and $8^{\circ}$ on nine different axis $(-45,-30,-20,-10,0$, $+10,+20,+30$, and +45 ) in the ipsilateral hemifield only (see Fig. $1 D$ ). It is important to note that for experiments 2 and 3, +ve and - ve refer to the upper and lower visual fields, respectively, and 0 indicates a distractor on the target axis. Subjects completed two blocks (left and right saccades) of 400 trials.

\section{RES ULTS}

Experiment 1 a targets at 4 and $8^{\circ}$, distractors presented along a horizontal axis in both visual fields

LATENCY. Mean saccade latency for an individual subject from experiment $1 a$ is plotted as a function of distractor eccentricity in Fig. 2. The results for the two blocks of trials (leftward and rightward saccades ) are plotted separately and appear symmetrical, showing that latency was not influenced by saccade direction. Saccade latency was increased (remote distractor effect) by some 30-40 ms, compared with the single target control situation, when a distractor appeared at fixation. Latency also was increased (by some $10-30 \mathrm{~ms}$ ) when distractors appeared in the contralateral hemifield. The inhibitory effect on latency increases as the contralateral distractors approach fixation. In contrast, saccade latency was unaffected by the appearance of a distractor at any eccentricity in the ipsilateral (target) hemifield.

There was no evidence of a directional latency asymmetry ( mean leftward $=162.6 \mathrm{~ms}$, mean rightward $=164.3 \mathrm{~ms}$ ), and the data for the two directions were collapsed together [absence of left/right differences was confirmed by analysis of variance (ANOVA), see next paragraph]. The data for the six subjects was pooled, and mean saccade latency is plotted in Fig. $3 A$ as a function of distractor eccentricity. Mean latency obtained to single targets at 4 and $8^{\circ}$ was 152 and $156 \mathrm{~ms}$, respectively, and the maximum latency was obtained when distractors appeared at fixation (193.5 ms). Latency was increased, compared with the single target (control) latency, when distractors appeared in the contralateral hemifield, but was not influenced by the appearance of a distractors in the ipsilateral hemifield.

A three-factor ANOVA compared saccade latency obtained in all conditions and revealed no effect of target direction $[F(1,5)=1.6 \mathrm{NS}]$, or target eccentricity $[F(1,5)=$ $5.6 \mathrm{NS}]$ on latency. A main effect was observed for distractor condition $[F(11,55)=32.4, P<0.0001]$. A post hoc analysis (Newman-Keuls) showed that latency was significantly increased (compared with single target latency) when distractors were presented at central fixation and with distractors at all locations in the contralateral hemifield (except at $\left.-10^{\circ}\right)$. Latency was unaffected by the presentation of a distractor in the ipsilateral (target) hemifield. A significant two-way interaction effect was revealed between target eccentricity and distractor location $[F(11,55)=3.9, P<$ 0.01]. The interaction resulted from a greater increase in latency with distractors in the contralateral hemifield for targets at $8^{\circ}$ than for targets at $4^{\circ}$.

AMPLITUDE. Mean saccade amplitude was cumulated for all subjects and collapsed for the two directions and is plotted in Fig. $3 B$. The modulation of saccade amplitude by distractors was the inverse to the effects observed on latency. Thus there was no effect on amplitude of distractors at fixation, or in the contralateral hemifield, but amplitude was influenced by the appearance of a distractor in the same hemifield along the same axis as the target. When the target appeared at $4^{\circ}$ and a distractor appeared more peripherally (at 6,8 , or $10^{\circ}$ ), a small increase in amplitude (global effect) was observed. Similarly amplitude increased when the target appeared at $8^{\circ}$ and a distractor appeared at $10^{\circ}$. In trials where the distractor appeared closer to the fovea than the $8^{\circ}$ target (at 2,4 , or $6^{\circ}$ ), then saccades appear to have been directed to the near distractor location and not to the target. The tendency to direct saccades to a near ipsilateral distractor may reflect the timing of the display sequence. It is plausible that the timing was insufficient to enable subjects to discriminate between the target and a near distractor, and so saccades were directed to the nearest stimulus as a default strategy. It is important to note that the latency of saccades directed to the more foveal distractors was no different to that obtained when a single target appeared in the control condition. Although saccade amplitude was modulated by the presence of an ipsilateral distractor, latency was not influenced. Saccade latency increased only when distractors appeared in
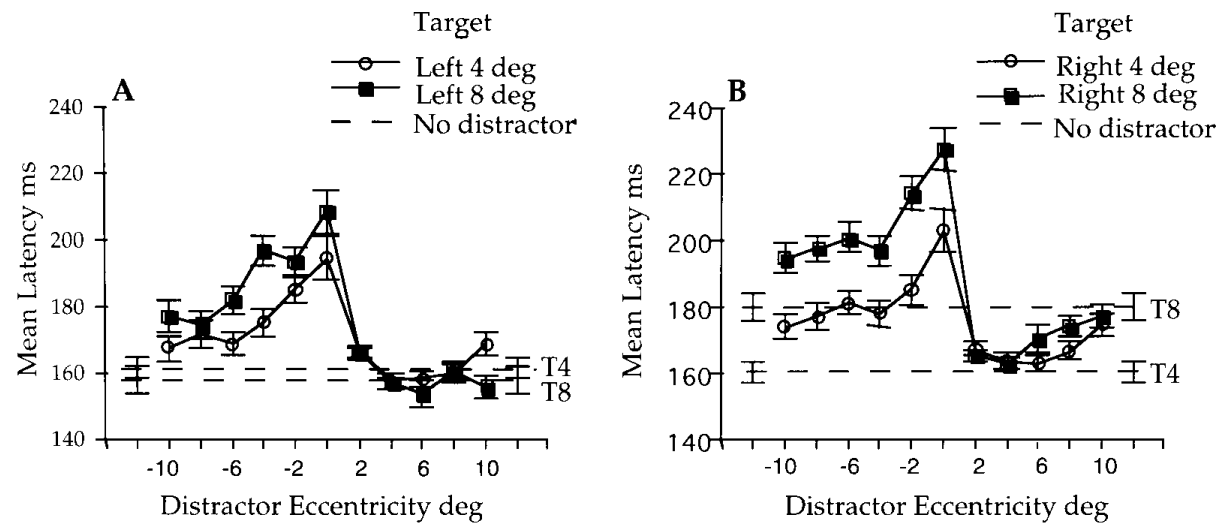

FIG. 2. Effects of remote distractors on saccade latency for a single subject in experiment 1a. $A$ : leftward saccades, $B$ : rightward saccades. Mean latency obtained to a single target (control condition) is shown as - - - (vertical lines indicate $1 \mathrm{SE}) . \circ$, targets at $4^{\circ}$ and $\mathbf{m}$, targets at $8^{\circ}$. Saccade latency was increased when distractors appeared at fixation $(0)$ or in contralateral hemifield ( - ve ) but was not influenced by distractors in ipsilateral (target) hemifield (+ve). Results for 2 saccade directions appear similar and reveal no evidence of directional latency asymmetries. 

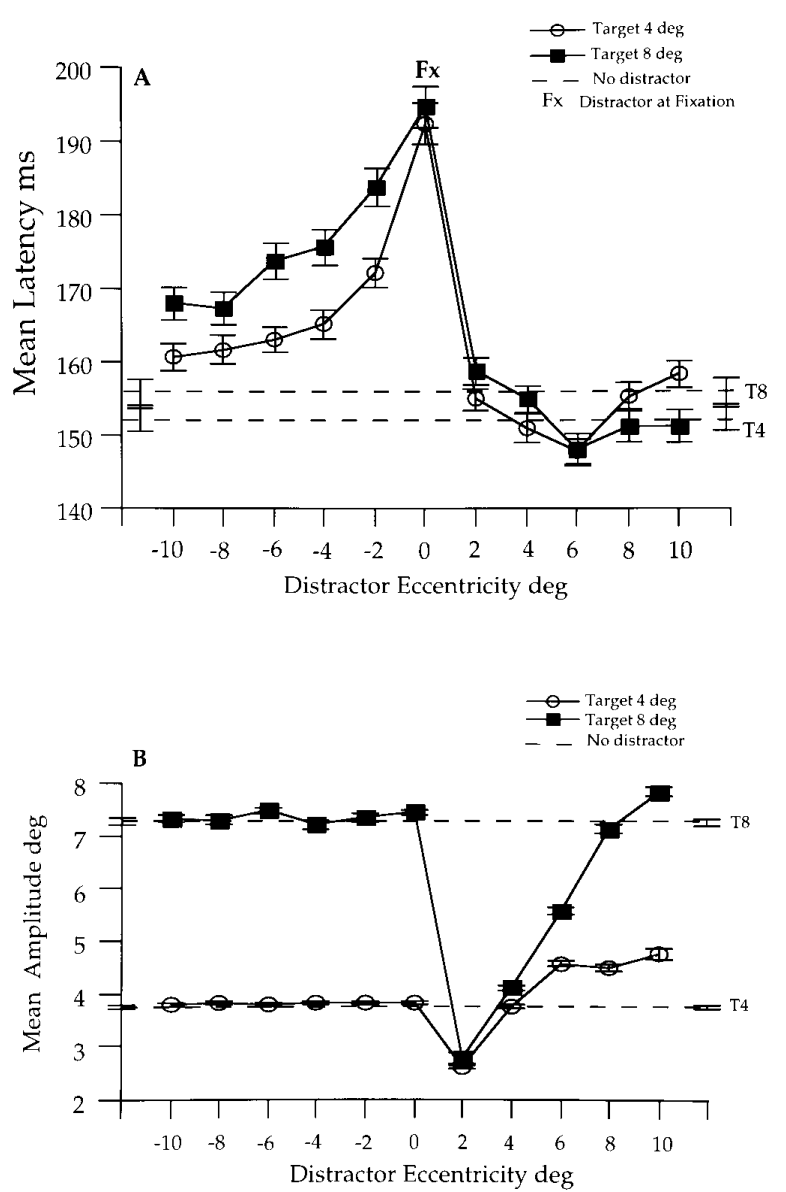

FIG. 3. Effects of remote distractors on saccade latency $(A)$ and amplitude $(B)$ pooled for all 6 subjects for experiment $1 a$. Data for 2 saccade directions (leftward and rightward) have been collapsed (error bars $=1$ $\mathrm{SE}$ ). - - - , mean latency obtained to single targets in control condition. $\bigcirc$ and $\mathbf{n}$, targets presented at 4 and $8^{\circ}$ eccentricities, respectively. Maximum latency was observed when a distractor appeared at fixation. Latency was also increased when a distractor appeared in contralateral hemifield $(-\mathrm{ve})$ but was not influenced by distractors in ipsilateral (target) hemifield (+ve). Saccade amplitude was not influenced by appearance of a distractor at fixation or in contralateral hemifield but was modulated when a distractor appeared in ipsilateral hemifield.

the contralateral hemifield, or at fixation, and under these conditions, saccade amplitude was comparable with that obtained to a single target.

\section{Experiment $1 b$ targets at $0.5,1,2$, and $4^{\circ}$, distractors presented along a horizontal axis in both visual fields}

LATENCY. In experiment $1 b$, targets were presented close to the central foveal region (eccentricities $0.5-4^{\circ}$ ) and distractors appeared at various eccentricities in either the ipsilateral or contralateral hemifields ( see Fig. $1 B$ ). The resulting latency distributions cumulated across all subjects are shown in Fig. 4 for one representative target eccentricity $\left(2^{\circ}\right)$. The histograms show latency distributions obtained to single targets or when distractors appeared at fixation or in the contralateral $\left(-1^{\circ}\right)$ or ipsilateral hemifield $\left(+4^{\circ}\right)$. Similar distributions with a single peak at $\sim 150-170 \mathrm{~ms}$ were obtained for the single target control condition as in the ipsilateral distractor condition. An overall shift in the latency distributions with a peak at $180-200 \mathrm{~ms}$ and an increase in the dispersion occurred when distractors appeared at fixation or in the contralateral hemifield.

Mean saccade latency was cumulated for all subjects and collapsed for the two blocks [ saccade direction was not significant $(F<1)]$ and is plotted in Fig. 5 as a function of distractor eccentricity. A two-factor ANOVA [target eccentricity (4), distractor eccentricity (8)] revealed a significant effect of target eccentricity $[F(3,15)=17.8, P<0.001]$. A post hoc analysis (Newman-Keuls) revealed no difference in mean latency for target eccentricities of 1,2 , or $4^{\circ}$, but a small additional latency increase was found for targets at $0.5^{\circ}$ eccentricity. The main effect of distractor eccentricity was significant $[F(7,35)=49.3, P<0.001]$, and a twoway interaction effect was observed $[F(21,105)=6.6$, $P<0.001]$. A post hoc analysis revealed that the mean latency obtained with distractors in the ipsilateral hemifield was comparable with that obtained to a single target. Latency was increased significantly when distractors appeared at fixation or at any location in the contralateral hemifield compared with the single target latency.

AMPLITUDE. The effects on saccade amplitude were similar to those observed in experiment $1 a$ in that amplitude was not influenced by the appearance of a distractor at fixation or at any location in the contralateral hemifield. When saccades were made to targets at $0.5,1$, and $2^{\circ}$ eccentricities and a distractor appeared more peripherally $\left(\right.$ at $\left.+4^{\circ}\right)$, a global effect increase in amplitude (of 17-19\%) was observed. The effects of distractors on amplitude and latency were reciprocal in nature. Ipsilateral distractors modulated amplitude if located more peripherally than the target but did not influence latency. Distractors presented at fixation or in the contralateral hemifield did not influence amplitude but did increase saccade latency.

\section{Experiment 2 targets at 4 and $8^{\circ}$, distractors presented at 2-D locations in both visual fields}

LATENCY. In experiment 2, the spatial-temporal relationship of the remote distractor effect was examined further by presenting distractors at various 2-D locations in both the ipsilateral and contralateral hemifields (Fig. $1 C$ ). The data were cumulated for all subjects and collapsed for two directions, and mean latency is plotted in Fig. $6 \mathrm{~A}$ as a function of distractor location. Saccade latency was generally slowest for targets at $8^{\circ}$ with distractors at $4^{\circ}$ and fastest for the targets at $4^{\circ}$-distractor at $8^{\circ}$ combinations. The greatest latency increase was obtained when distractors appeared at central fixation (188 ms). Saccade latency also was increased but to a lesser degree, when distractors appeared at all locations except when presented on the ipsilateral target axis.

A three-factor ANOVA [ target eccentricity (2), distractor eccentricity (2), distractor axis (8)] compared the magnitude of the effects on latency when distractors appeared at various 2-D locations. The comparison excluded distractors at fixation as the factor of distractor axis is not applicable in this instance. The main effect of target eccentricity was significant $[F(1,6)=7.3, P<0.05]$, indicating that saccade latency was faster for targets at $4^{\circ}$ than at $8^{\circ}$. The main effect of distractor eccentricity was significant $[F(1,6)=19.8$, 

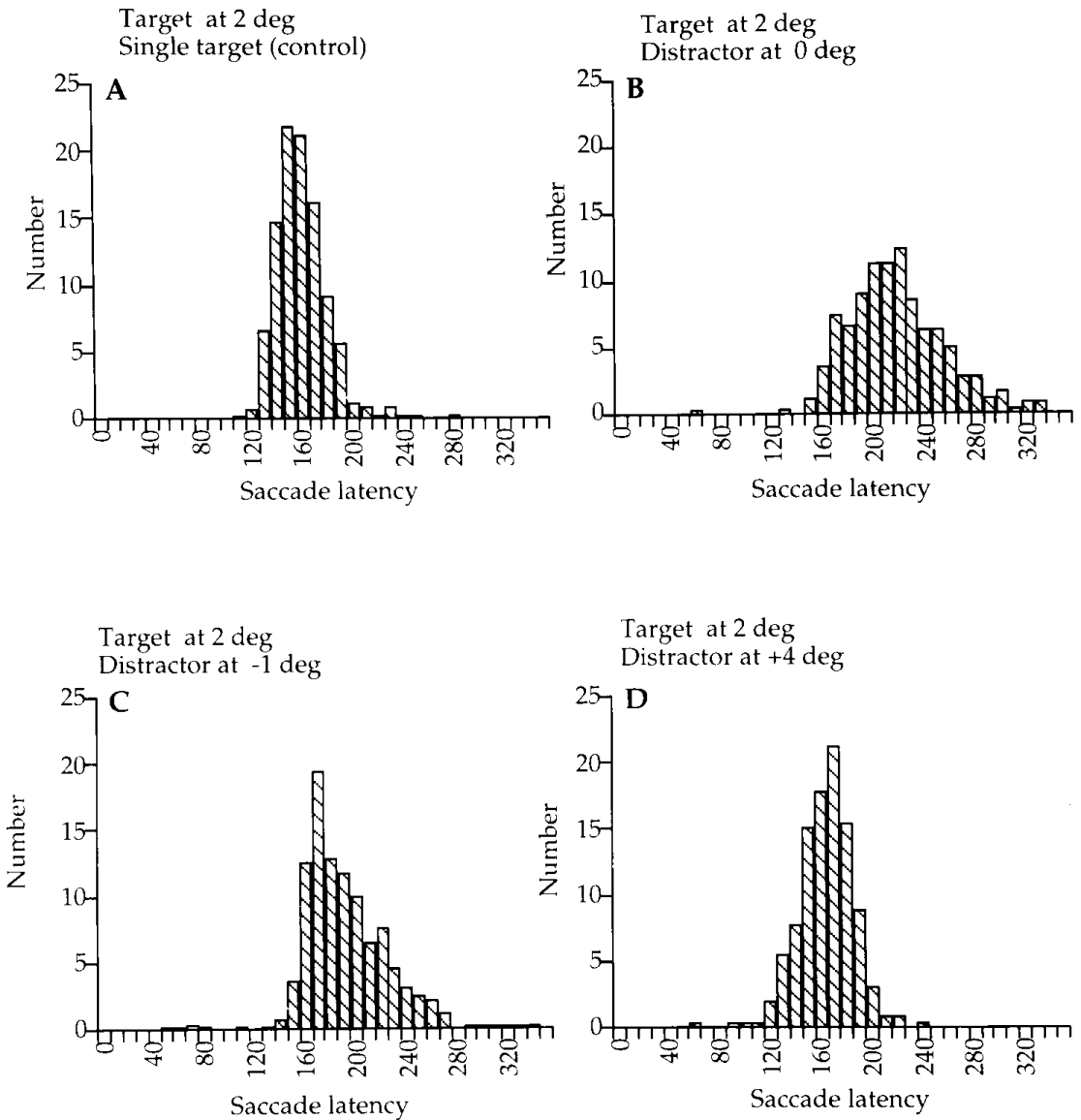

FIG. 4. Histograms of distributions of saccade latency (binwidth $10 \mathrm{~ms}$ ) obtained in experiment $1 b \mathrm{cu}$ mulated for all subjects. Saccades were made under single target control conditions $(A)$, when a distractor appeared at fixation $(B)$, in contralateral hemifield at $-1^{\circ}$ eccentricity $(C)$, or in ipsilateral target hemifield at $+4^{\circ}$ eccentricity $(D)$.
$P<0.001$ ], showing that a greater latency increase occurred with distractors presented at the $4^{\circ}$ eccentricity locations. The main effect of distractor axis $[F(7,42)=7.5, P<0.01]$ was also significant. Post hoc analysis (Newman-Keuls)

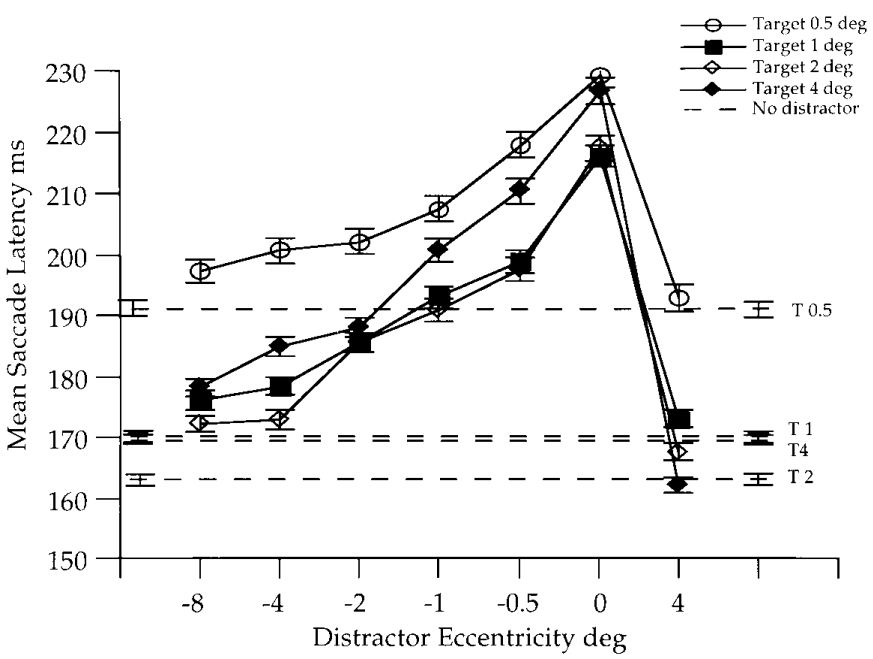

FIG. 5. Effects of remote distractors on saccade latency for experiment $1 b$. Data for 2 saccade directions (leftward and rightward) have been collapsed and pooled for all subjects (error bars $=1 \mathrm{SE}$ ). --- , mean latency obtained to single targets in control condition. Symbols show latency observed when a targets were presented at eccentricities of $0.5,1,2$, and $4^{\circ}$. Latency was increased compared with control condition when a distractor appeared at fixation $(0)$ or in contralateral hemifield $(-v e)$ but was not influenced by a distractor in ipsilateral (target) hemifield. showed that the latency obtained with distractors at any position other than on the target axis differed from that obtained with distractors along the target axis. There was no difference among the seven other axes, showing that the distractor effect latency increase was comparable for all off axis distractor locations. A significant three-way interaction effect also was revealed $[F(7,42)=2.8, P<0.05]$. It can be seen from Fig. $6 A$ that when the target was at $4^{\circ}$ and the distractor eccentricity was $8^{\circ}$, there was very little influence of distractor location. However, for targets and distractors at $8^{\circ}$, there was an influence of distractor position on latency. The latency obtained with distractors at the target location (0) was similar for both target and distractor eccentricity combinations.

AMPLITUDE. A dissociation was again observed between the effects on latency and on amplitude dependent on the spatial location of distractors. Mean saccade amplitude is plotted in Fig. $6 B$ and shows that amplitude largely was unaffected by the presentation of a distractor away from the target axis, the exception being the undershoot of the $8^{\circ}$ target location when distractors appeared at $4^{\circ}$ eccentricities at positions of $\pm 45^{\circ}$ away from the target axis. With targets at $8^{\circ}$ and distractors at $4^{\circ}$ on the ipsilateral target axis, subjects appeared to direct their saccades to the near distractor location. An increase in amplitude (global effect) was observed for targets at $4^{\circ}$ with distractors at $8^{\circ}$ on the horizontal target axis. Distractors located on the ipsilateral target axis modulated saccade amplitude but did not affect latency. Distractors presented away from the target axis in both the contralateral 
and ipsilateral hemifields increased latency without modulating amplitude.

\section{Experiment 3 targets at 4 and $8^{\circ}$, distractors presented in} the ipsilateral visual field

LATENCY. In experiment 3 , targets and distractors were presented at eccentricities of 4 and $8^{\circ}$, and in some trials, distractors appeared at central fixation or on axis of $0, \pm 10$, $\pm 20, \pm 30$, or $\pm 45^{\circ}$ in the ipsilateral hemifield (Fig. $1 D$ ). Mean saccade latency cumulated for all subjects and collapsed for the two directions is plotted in Fig. 7A. The maximum latency $(190 \mathrm{~ms})$ was observed when distractors appeared at fixation. Distractors presented on axes within $\pm 20^{\circ}$ of the target axis appear to have had little effect on saccade latency, but when distractors appeared on axis $>20^{\circ}$ from horizontal, latency was increased by some $10-20 \mathrm{~ms}$. A three-factor ANOVA confirmed that latency was significantly faster for the $4^{\circ}$ eccentricity location $[F(1,6)=33$, $P<0.001]$ and that distractors at $8^{\circ}$ produced less of an
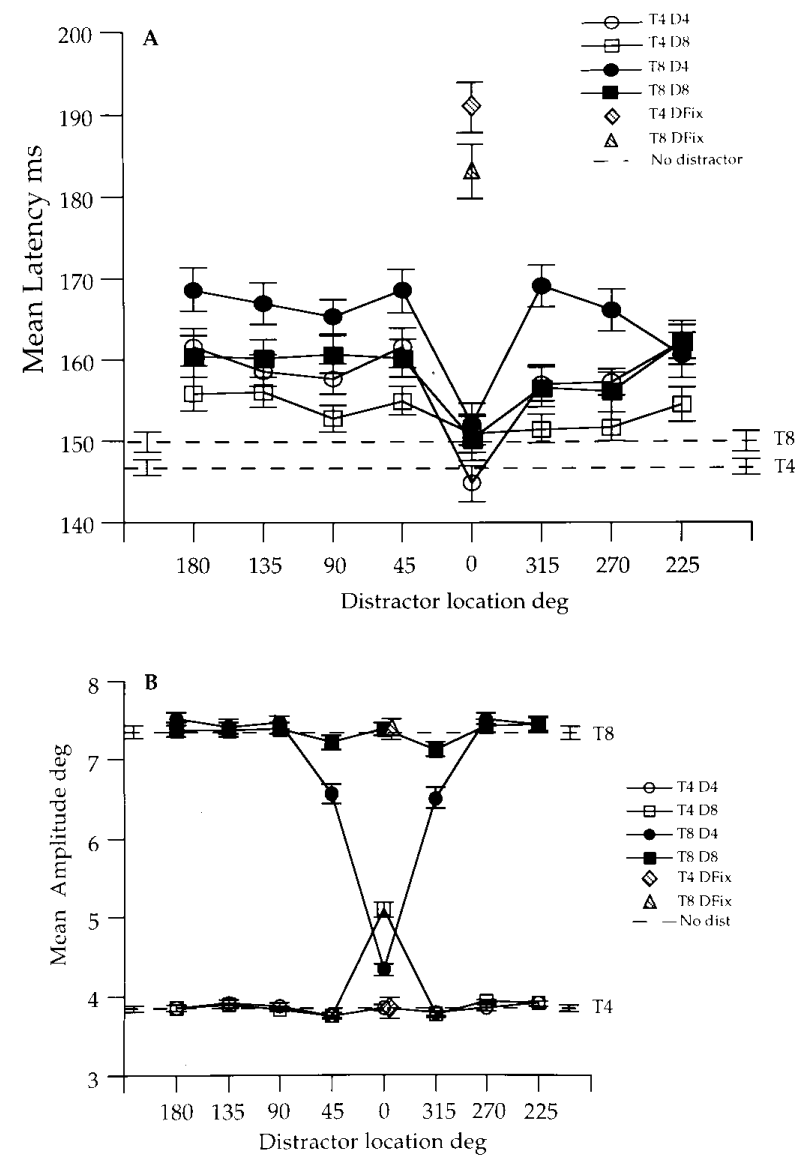

FIG. 6. Effects of remote distractors presented at 2-dimensional locations, on saccade latency $(A)$ and amplitude $(B)$ for experiment 2. Data have been pooled for all subjects and collapsed for 2 saccade directions (leftward and rightward). - - - , mean latency obtained to single targets in control condition. Open and closed symbols are for targets presented at 4 and $8^{\circ}$ eccentricities, respectively (error bars $=1 \mathrm{SE}$ ). Maximum latency was observed when distractors appeared at fixation. Latency also was increased when distractors appeared at all locations in both visual fields except when on ipsilateral target axis. Saccade amplitude was not influenced by a distractor at fixation or by distractors presented away from target axis but was modulated when a distractor appeared on ipsilateral target axis.
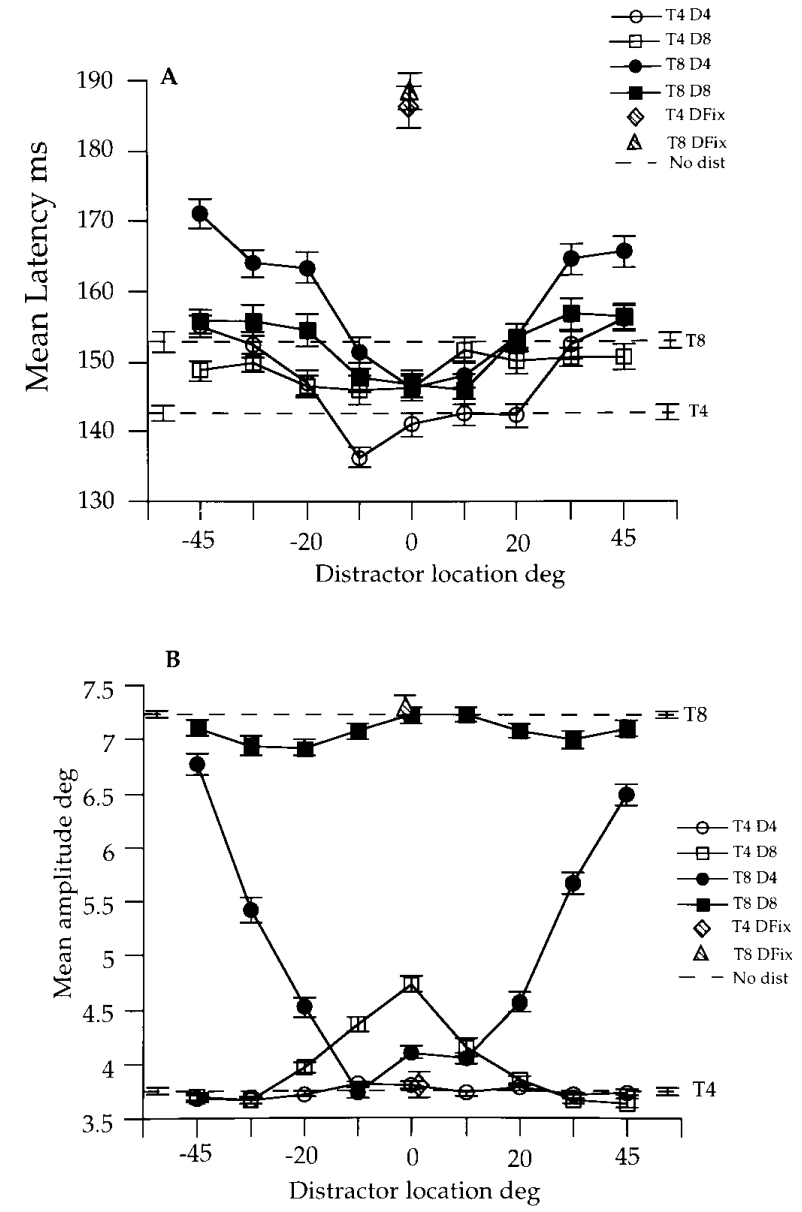

FIG. 7. Effects of ipsilateral distractors presented within $\pm 45^{\circ}$ of horizontal target axis on saccade latency $(A)$ and amplitude $(B)$ in experiment 3. Data have been pooled for all subjects and collapsed for 2 saccade directions (leftward and rightward). - - - , mean latency obtained to single targets in control condition. Open and closed symbols are for targets presented at 4 and $8^{\circ}$ eccentricities, respectively (error bars $=1 \mathrm{SE}$ ). Maximum latency was observed when distractors appeared at fixation. Distractors presented on axis $>20^{\circ}$ from target axis increased saccade latency. Distractors presented within $20^{\circ}$ of target axis did not influence latency but did modulate amplitude.

effect on latency than did distractors located at $4^{\circ}$ eccentricities $[F(1,6)=6.5, P<0.05]$. The main effect of distractor axis was highly significant $[F(8,48)=18, P<0.0001]$. A post hoc analysis (Newman-Keuls) showed that latency obtained when distractors were presented on the horizontal target axis was comparable with that obtained with distractors positioned on an axis $10^{\circ}$ away. Latency, however, was increased significantly when distractors were positioned on axis 20,30 , and $45^{\circ}$ away from the horizontal target axis $(P<0.01)$.

A significant two-way interaction effect was observed for target eccentricity by distractor position $(P<0.001)$. The difference in latency for the two target eccentricities was smaller for distractors positioned $<20^{\circ}$ of the target axis than was observed when distractors appeared at positions $>20^{\circ}$ from the target axis (in which case a greater influence on latency was observed for targets at $8^{\circ}$ than was observed for targets at $4^{\circ}$ ). A significant interaction effect also was observed for distractor eccentricity by distractor position 
$(P<0.001)$. Distractors presented at eccentricities of $4^{\circ}$ produced a greater increase in latency at positions $>20^{\circ}$ and a smaller effect at positions $<20^{\circ}$ of the target axis than did distractors at $8^{\circ}$ eccentricities.

AMPLITUDE. Figure $7 B$ shows the effects of distractors on saccade amplitude. A global effect amplitude modification was observed when distractors appeared at eccentricities of $8^{\circ}$ positioned within $\pm 20^{\circ}$ of the horizontal target axis. Distractors presented at the same eccentricity as the target did not substantially modulate amplitude. When the target appeared at $8^{\circ}$ and distractors appeared more foveally at $4^{\circ}$, then subjects appeared to direct their saccades toward the near distractor location. It is important to note that the reciprocal relationship between amplitude modulation and latency increase again was observed. Distractors presented on the target axis or on axis within $20^{\circ}$ of the target axis modulated amplitude but did not increase latency. Distractors presented at fixation or on axis $>20^{\circ}$ from the target axis had no effect on amplitude but did increase latency.

\section{Relationship between the remote distractor effect and the ratio of distractor to target eccentricity}

The present series of experiments have revealed a robust and systematic increase in saccade latency after the appearance of a distractor at various locations in both the ipsilateral and contralateral visual fields. The greatest latency increase was observed for distractors at central fixation. However, the appearance of a distractor at nonfoveal locations in either visual field also increased saccade latency, the only exception being when distractors appeared in the ipsilateral hemifield within about $\pm 20^{\circ}$ of the target axis. A detailed examination of the relationship between distractor location and magnitude of the remote distractor effect was performed on the data from all four experiments. The magnitude of the remote distractor effect was calculated by subtracting the mean latency obtained in trials in which distractors appeared from the single target mean. As already mentioned, the latency increase was not observed when distractors appeared within $20^{\circ}$ of the ipsilateral target axis, and so the effects on latency were examined only for conditions in which distractors appeared on axis $\pm 45, \pm 90, \pm 135$, and $\pm 180^{\circ}$ away from the horizontal target axis. Conditions in which distractors appeared on the ipsilateral target axis $\left(0^{\circ}\right)$ or on axis within $45^{\circ}$ of the target axis (experiment 3 only) were not included. Figure $8 \mathrm{~A}$ shows the combinations of target eccentricities and distractor locations that were included to examine the effects of distractors on latency. The remote distractor latency increase is plotted as a function of the ratio of distractor to target eccentricity in Fig. $8 B$.

Figure $8 B$ shows that the increase in saccade latency depends in a highly systematic way on the relationship between target and distractor eccentricities. The greatest slowing occurs for distractors at central fixation. The points obtained for other distractor locations lie on a smooth function with the latency increase found with foveal distractors forming part of the same function. Thus under these experimental conditions, the magnitude of the latency increase is entirely predictable by the ratio of distractor to target eccentricity, and the difference between foveal and nonfoveal distractors is quantitative rather than qualitative.
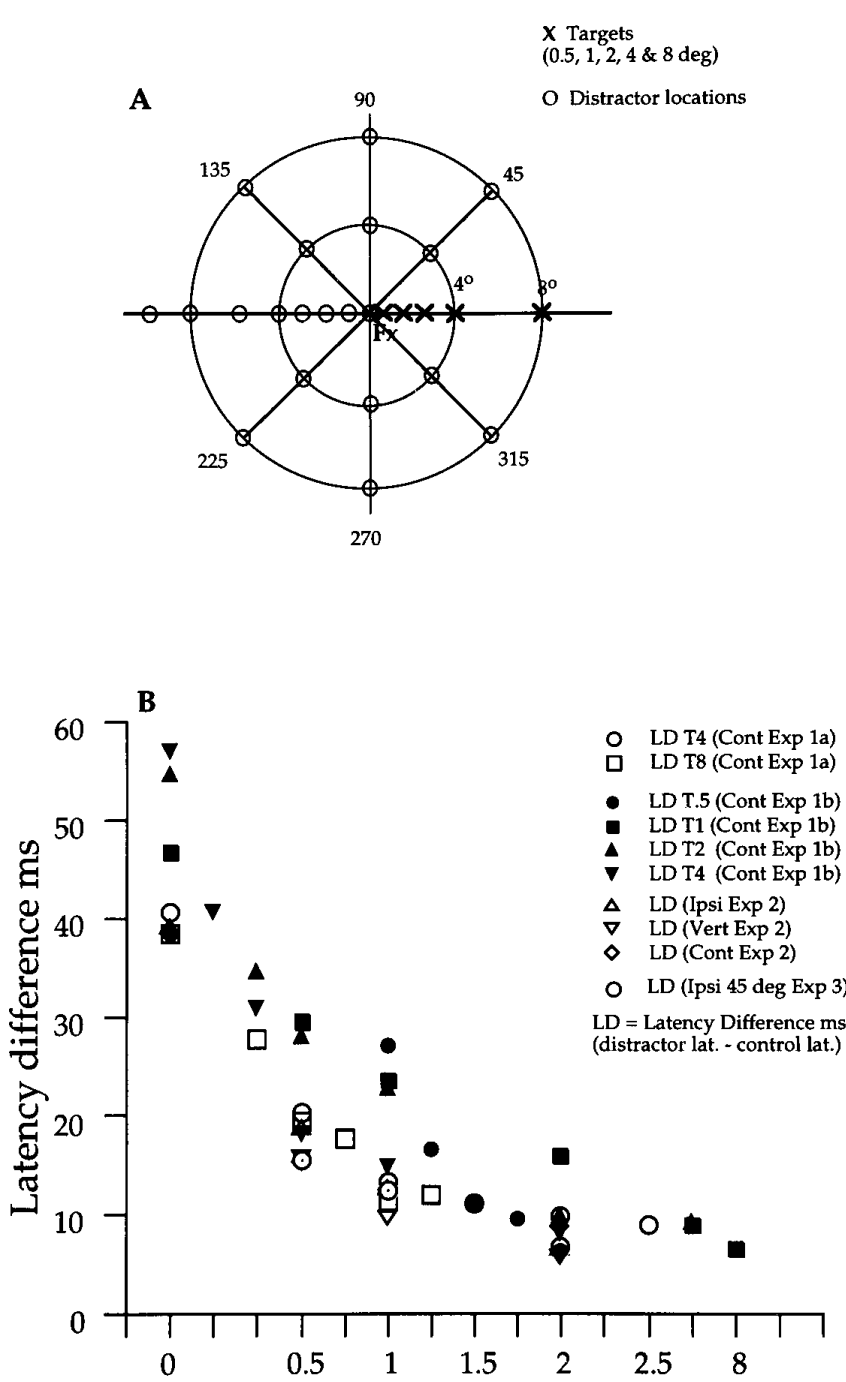

Ratio distractor to target eccentricities

FIG. 8. A: schematic representation of target (X) and distractor (0) locations used to calculate latency difference LD ( $L D=$ distractor latency single target latency) plotted as a function of ratio of distractor to target eccentricities in $B$. A ratio of 0 indicates a distractor presented at fixation; a ratio of 1 indicates target and distractor at equal eccentricities (e.g., $4 / 4^{\circ}, 8 / 8^{\circ}$ ), etc. Effects of distractors presented on axis $\pm 45, \pm 90, \pm 135$, $\pm 180^{\circ}$ away from target axis in ipsilateral and contralateral hemifield for all 4 experiments were considered. For experiments $1 a$ and $1 b$, data for different target eccentricities are plotted separately under conditions in which distractors appeared at fixation or on the contralateral horizontal axis. In experiments 2 and 3, data were collapsed for 2 target eccentricities. Data points for 2 oblique axes in ipsilateral hemifield (Ipsi), 2 vertical axes (Vert), and 3 contralateral axes (Cont) were averaged and plotted separately. $B$ : latency difference (remote distractor effect) plotted as a function of ratio of distractor to target eccentricities. Plot shows that maximum increase in saccade latency was observed when a distractor appeared at fixation but the increase for other distractor locations depends in a highly systematic way on ratio of distractor to target eccentricities.

\section{DISCUSSION}

The present series of experiments, in confirmation of earlier work (Walker et al. 1995; Weber and Fischer 1994) has shown that the onset of a simultaneous distractor stimulus at a nontarget location produces a robust increase in the latency of a saccade to a visual target (the remote distractor effect). The increase in latency occurred with distractors at 
any location in the visual field with the exception of those in a narrow sector of $\sim 40^{\circ}$ width centered on the target axis. Distractors inside this sector modify the amplitude of the saccade but do not affect its latency. Outside this sector, a distractor in either an ipsilateral or a contralateral position produced an increase in the latency of the saccade. There was no suggestion that the latency increase was any different when the distractor was presented at the mirror symmetrical location to the target or at locations on the axis opposite to the target. Although saccade latency was increased generally when the target appeared at small eccentricities within the so called "dead-zone" (Kalesnykas and Hallett 1994; Weber et al. 1992), the remote distractor effect again was observed with targets at an eccentricity of $0.5^{\circ}$.

The magnitude of the latency increase produced by a remote distractor was found to decrease monotonically as the distractor was positioned more peripherally. The maximum increase in saccade latency was observed when the distractor appeared at central fixation ${ }^{1}$. The latency increase observed when distractors appeared at other locations in both visual fields fitted the same continuous function including the points obtained for distractors at fixation (Fig. 8). This function reveals that the magnitude of the remote distractor latency increase depends in a highly systematic way on the ratio of distractor to target eccentricity. There is, therefore, no qualitative, but only a quantitative, difference between the inhibitory effects observed at fixation from those operating in other regions of the visual field. It has been suggested that the increase in latency of a distractor at fixation may be accounted for in terms of increased activation of a fixation region (Dorris and Munoz 1995; Munoz and Wurtz 1992, 1993a,b, 1995a,b) that inhibits the triggering of the saccade. Our behavioral findings therefore imply that this inhibitory process operates over a wide region of the visual field.

Our results revealed a reciprocal relationship between the effects observed on latency and amplitude; distractors in remote positions increase latency but not amplitude, whereas distractors in neighboring positions influence amplitude but not latency (see Fig. 9). The results show a "window" centered on the target and extending over axes within about $\pm 20^{\circ}$ of the target axis within which amplitudes, rather than latencies, are affected. There is then quite a sharp transition to the opposite effect with distractors located anywhere outside this window producing the remote distractor latency increase. The reciprocal nature of the latency and amplitude

${ }^{1}$ In all the experiments reported here, a "0 gap"' condition was used whereby fixation offset occurred simultaneously with the onset of the target and distractor. It could be argued that a distractor appearing at fixation is a stimulus "transition", and not a novel event (as is the case for a peripheral distractor). It is plausible to suggest, therefore, that the effects on saccade latency for an event at fixation may be different to those observed for peripheral distractors because it is a stimulus transition. Although accepting this possibility, we note that in our experiments the appearance of a distractor at fixation was not a simple transition as the fixation stimulus was a small cross (width $0.19^{\circ}$ ), whereas distractors were larger open circles $\left(0.53^{\circ}\right)$ in diameter. A study directly relevant to this issue has been performed by Ross and Ross (1980) who assessed the effects on saccade latency of stimulus onsets and change at fixation, and they found that the effects were indistinguishable. This finding supports our view that the greater effects on latency for distractors at fixation are not because stimulus transitions have stronger inhibitory effects. We are grateful to an anonymous referee for highlighting this issue.

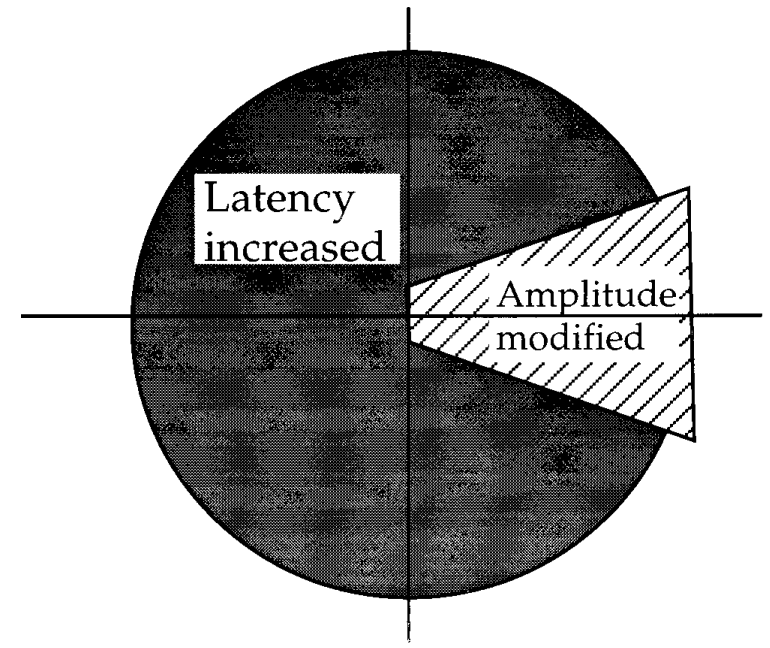

FIG. 9. Schematic diagram of visual field illustrating the reciprocal effects on saccade latency and amplitude depending on distractor location. Distractors presented within a window of $20^{\circ}$ around target axis modulate amplitude but did not influence latency. Distractors presented away from target axis $\left(>20^{\circ}\right)$ increase latency but had no effect on amplitude.

effects are suggestive of inhibitory and facilitative processes operating within the oculomotor system. Stimuli in close proximity appear to be combined to form a single peak of activity with no increase in latency. Stimuli located outside this window appear to be encoded as separate peaks but at a cost of an increase in saccade latency.

The modulation of saccade amplitude occurs when distractors appear within a narrow sector of $\pm 20^{\circ}$ around the saccade meridian. This finding is consistent with previous work on saccadic adaptation (Deubel 1987, 1991) and from studies of the global effect (Deubel et al. 1984; Findlay 1982). Deubel (1987) showed that both saccade gain and direction may be modulated after an adaptation phase where the target stimulus is displaced during the intersaccadic interval. Furthermore, the adaptation effects were found to be limited to saccades elicited within $30^{\circ}$ of the adapted direction. The model proposed to account for the adaptation effects was based on a sensory motor map containing motor channels of $\sim 30^{\circ}$ in width. Stimuli located within the $30^{\circ}$ receptive field contribute to the vectorial amplitude signal after a weighted integration of the input signal. Stimuli located further away from the target direction had less influence on the computation of the saccade vector than did stimuli close to the target. The spatial averaging process has been linked directly to processes of distributed population coding operating within the oculomotor system. Glimcher and Sparks (1993) examined the neural substrate of averaging saccades and showed that a single population of burst neurons was active before the saccade. Thus the modulation of saccade amplitude may be a consequence of the mode of operation of the collicular burst neurons where two stimuli can produce a single intermediate peak of activity. Our findings have confirmed that this spatial averaging process is restricted to stimuli located within $20^{\circ}$ from the target axis.

The increase in saccade latency observed with distractors in both visual fields can be accounted for in terms of inhibitory mechanisms operating in neural structures involved in saccade programming. A report by Weber and Fischer 
(1994) revealed that short-latency express saccades were almost absent when distractors appeared closer than $2^{\circ}$ of the fovea. The numbers of express saccades also were reduced when distractors appeared in either the contralateral or the ipsilateral visual field at eccentricities as far out as $12^{\circ}$. The presence of inhibitory effects continuing beyond the fixation location as described in the present study extends our understanding of the processes of response competition. The exact nature of the neurophysiological correlate of this relationship is not yet clear, however. The superior colliculus is one structure known to be involved in the generation of saccades (Schiller et al. 1980), and neurons in the intermediate layers are thought to form a motor map that codes the amplitude and direction of the desired saccade (McIlwain 1986; Sparks 1986; Sparks and Hartwich-Young 1989). We consider two ways in which the effects we have demonstrated might relate to processing in the superior colliculus. Our model is consistent with recent reports of inhibitory properties of collicular neurons and provides one plausible explanation of the remote distractor effect. It should be noted that saccade generation involves a circuit of cortical and subcortical structures (see Fischer 1987; Pierrot-Deseilligny et al. 1991 for reviews), and we acknowledge that similar processes also may be observed in other brain regions involved in saccade generation.

In a previous paper on saccade programming (Walker et al. 1995), we suggested that inhibitory processes might operate directly between the location of the superior colliculus representing the spatial location of the target and that representing the location of the distractor. Reciprocal inhibition of this sort could provide the basis for a competitive inhibition process reflecting target selection. Intracollicular inhibitory mechanisms have been found to operate within the colliculus of the cat (Rizzolatti et al. 1974) and monkey (Wurtz et al. 1980). When the distractor is in the opposite hemifield, similar intercollicular inhibitory processes would have to operate via crossed inhibitory projections.

There are several difficulties in attributing the remote distractor effect to such direct inhibitory processes. First, it would be likely that the effect would show a direct dependence on the distance between target and distractor locations rather than the relationship actually found. The strength of the effect with contralateral distractors is also a problem. There is evidence for crossed collicular inhibition (Infante and Leiva 1986; Sprague 1966; Wurtz et al. 1980) but its effects are weaker than those within one colliculus. Additionally, such influences presumably would be delayed because of the time required for inhibitory signals to cross the collicular commissure or other commissural pathways (Wallace et al. 1989, 1990). Such a time delay would make it unlikely that the effects on latency observed for distractors in the opposite hemifield would fit the same function as with distractors in the same hemifield.

A second, alternative, explanation is suggested by the continuity of the remote distractor effect between foveal and nonfoveal locations. Significant progress recently has been made in relating visual events at fixation concerned with saccade control to physiological processes within the superior colliculus. Munoz and Wurtz (1992, 1993a,b) have shown that the rostral pole region of the colliculus (that represents the central $2^{\circ}$ of the visual field) contains neurons that respond during active fixation. Cells in this region show a tonic discharge during periods of fixation. The temporary deactivation of these neurons (Munoz and Wurtz 1993b) reduced the animals' ability to maintain fixation, increased the numbers of (erroneous) express saccades made, and reduced saccade latency. These findings are interpreted as showing that the rostral region operates as an ocular fixation mechanism. There appear to be cross-connections between the rostral pole regions in each colliculus so that the system works largely as a single unit.

Munoz and Wurtz (1995ab) extended their findings and identified three main types of collicular neurons on the basis of their presaccadic activity. These were termed: fixation cells, buildup cells, and burst cells. Fixation cells were localized in the rostral pole region and showed a sustained response to a fixation stimulus. Buildup cells and burst cells were located throughout the rest of the collicular map. Buildup cells showed a gradual rise in activity after the onset of a peripheral saccade target that was related reciprocally to activity in fixation cells; buildup cell activity increased as fixation cell activity decreased. In contrast, burst cells showed a sudden burst of activity just before saccade onset. Munoz and Wurtz suggest that the buildup cells are involved in the preparation to make a saccade, whereas burst cells may encode the metrics of the desired movement. There are, therefore, two ways in which the fixation mechanism is thought to inhibit the production of a saccade. First, it is thought to inhibit the activity of neurons in other regions of the colliculus and, second, it is thought to excite the brain stem omnipause neurons (Paré and Guitton 1994). If fixation cell activity operates at the level of the brain stem omnipause neurons, then distractors presented in either hemifield would have comparable inhibitory effects because the omnipause neurons do not form a lateralized system. The strength of the distractor effect with ipsilateral distractors was found to be similar to that observed with contralateral distractors, and this can be accounted for by the fixation activity operating at the brain stem level without recourse to crossed collicular inhibition.

Inhibitory collicular mechanisms have been incorporated into models of the gap effect. The gap effect refers to the decrease in saccade latency (first observed by Saslow 1967), which occurs when the central fixation stimulus is removed before the onset of the saccade target. The latency reduction observed under gap conditions is a robust finding that has been studied extensively in the monkey (Boch and Fischer 1986; Boch et al. 1984; Fischer and Boch 1983; Paré and Munoz 1996) and man (Fischer and Ramsperger 1984; Kingstone and Klein 1993; Mayfrank et al. 1986; ReuterLorenz et al. 1991, 1995; Ross, L. E., and Ross 1980; Ross, S. M., and Ross 1981; Walker et al. 1995). Detailed models of the gap effect have proposed that such a fixation mechanism could increase the time required to reach peak activation for cells in other regions of the motor map (Dorris and Munoz 1995; Munoz and Wurtz 1995a,b). The postulate here (with some experimental support) (Munoz and Wurtz 1993a) is that the activity of neurons in the fixation region, that is the rostral pole of the colliculus, is influenced directly by visual events at fixation. Visual offset decreases activity and renders saccade triggering more likely.

The converse postulate, that visual onset at fixation in- 
creases rostral pole activity and renders saccade triggering less likely, could offer a straightforward explanation of the increased latency found in the current study when distractors were at fixation. The results also have demonstrated that the remote distractor effect in nonfoveal regions shows continuity with the effect with foveal distractors. It thus is tempting to try to extend the explanation and argue that in all cases the remote distractor effect is caused by the effect of the distractor on the fixation system.

One plausible suggestion is that fixation cells are found at locations further from the fovea than the $2^{\circ}$ region of the rostral pole region. Supportive evidence for this view has been provided by Gandhi and Keller (1995), who reported that neurons resembling fixation neurons do extend to more caudal regions of the SC. Furthermore, Gandhi and Keller showed that stimulation of such fixation cells located at sites that are associated with saccades of amplitude $\leq 10^{\circ}$ can interrupt eye movements in midflight, whereas stimulation beyond the $10^{\circ}$ did not. The implication of this finding is that the fixation region may extend to $\sim 10^{\circ}$ from the fovea and that neurons throughout this area may inhibit saccade activity by projections to the omnipause neurons.

One of the striking aspects of our results is the relationship with the distractor to target eccentricity ratio observed. If the effect depended on distractor activation of the fixation region and the magnitude of this activation decreased as the distractor was positioned more peripherally, the diminishing effect of more peripheral distractors might be accounted for. Such a relationship seems plausible; for example, one explanation could be that the numbers of fixation cells decreases for more caudal locations within the SC. However, as the latency increase also was related to target eccentricity this cannot be the whole explanation. One possibility is that the inhibitory effects on buildup neurons become weaker as targets move further into the periphery.

We gratefully acknowledge the assistance of S. Hieke for running the experiments and I. Paprotta for further assistance. We also thank T. Hodgson and two anonymous referees for helpful criticisms on an earlier draft.

This work was funded by a grant awarded by the Max-Planck Gesellschaft.

Address for reprint requests: R. Walker, Dept. of Psychology, Royal Holloway, University of London, Egham, Surrey TW20 0EX, UK.

Received 18 November 1996; accepted in final form 29 April 1997.

\section{REFERENCES}

BECKER, W. AND JURgens, R. An analysis of the saccadic system by means of double-step stimuli. Vision Res. 19: 967-983, 1979.

Boch, R. AND FISCHER, B. Further observations on the occurrence of express-saccades in the monkey. Exp. Brain Res. 63: 487-494, 1986.

Boch, R., Fischer, B., AND RAMSPERGER, E. Express-saccades of the monkey: reaction time versus intensity, size, duration, and eccentricity of their targets. Exp. Brain Res. 55: 223-231, 1984.

Crane, H. D. And Steele, C. M. Generation-V dual Purkinje-image eyetracker. Appl. Optics, 24: 527-537, 1985.

Deubel, H. Adaptivity of gain and direction in oblique saccades. In: Eye Movements: From Physiology to Cognition, edited by J. K. O'Regan, and A. Lévy-Schoen. North Holland: Elsevier, 1987, p. 181-190.

Deubel, H. Plasticity of metrical and dynamic aspects of saccadic eye movements. In: Tutorials in Motor Neuroscience, edited by J. Requin and G. E. Stelmach. Amsterdam: Kluwer Academic, 1991, p. 563-579.

Deubel, H. AND BRidgeman, B. Fourth Purkinje image signals reveal eyelens deviations and retinal image distortions. Vision Res. 35: 529-538, 1995.
Deubel, H., Wolf, W., And Hauske, G. The evaluation of oculomotor error signals. In: Theoretical and Applied Aspects of Oculomotor Research, edited by A. G. Gale and F. Johnson. Amsterdam: Elsevier, 1984, p. 55-62.

Dorris, M. C. AND MunOz, D. P. A neural correlate for the gap effect on saccadic reaction times in monkeys. J. Neurophysiol. 73: 2558-2562, 1995.

FINDLAY, J. M. Global processing for saccadic eye movements. Vision Res. 22: $1033-1045,1982$.

FINDLAY, J. M. Visual information processing for saccadic eye movements. In: Spatially Oriented Behavior, edited by A. Hein and M. Jeannerod. New York: Springer-Verlag, 1983, p. 281-303.

FINDLAy, J. M. Visual computation and saccadic eye movements. Spatial Vision. 2: 175-189, 1987.

FISCHER, B. The preparation of visually guided saccades. Rev. Physiol. Biochem. Pharmacol. 106: 1-35, 1987.

Fischer, B. AND Boch, R. Saccadic eye movements after extremely short reaction times in the monkey. Brain Res. 260: 21-26, 1983.

FISCHER, B. AND RAMSPERGER, E. Human express saccades: extremely short reaction times of goal directed eye movements. Exp. Brain Res. 57: 191195, 1984.

Fischer, B. AND BREITMEYER, B. Mechanisms of visual attention revealed by saccadic eye movements. Neuropsychologia. 25: 73-83, 1987.

Fischer, B., Weber, H., Biscaldi, M., Aiple, F., Otto, P., and Stuhr, V. Separate populations of visually guided saccades in humans: reaction times and amplitudes. Exp. Brain Res. 92: 528-541, 1993.

GANDHI, N. J. AND KELLER, E. L. Interrupting saccades by electrical stimulation of the superior colliculus determines an extended fixation zone. Soc. Neurosci. Abstr. 21: 468.2, 1995.

GLIMCHER, P. W. AND SPARKS, D. L. Representation of averaging saccades in the superior colliculus of the monkey. Exp. Brain Res. 95: 429-435, 1993.

INFANTE, C. AND LEIVA, J. Simultaneous unitary neuronal activity in both superior colliculi and its relation to eye movements in the cat. Brain Res. 381: 390-392, 1986.

KalesnyKas, R. P. And Hallett, P. E. Retinal eccentricity and the latency of eye saccades. Vision Res. 34: 517-531, 1994.

Kingstone, A. And KLEIn, R. M. Visual offsets facilitate saccadic latency: Does predisengagement of visuospatial attention mediate this gap effect. J. Exp. Psychol. Hum. Percept. Perform. 19: 1251-1265, 1993.

LÉVY-SCHOEN, A. Determination et latence de la reponse oculomotrice a deux stimulus. L'Anee Psychol. 74: 43-66, 1969.

LÉVY-SCHOEN, A. AND BlaNC-GARIN, J. On oculomotor programming and perception. Brain Res. 71: 443-450, 1974.

LynCH, J. C. AND MCLAREN, J. W. Deficits of visual attention and saccadic eye movements after lesions of parietooccipital cortex in monkeys. $J$. Neurophysiol. 61: 74-90, 1989.

Mayfrank, L., Mobashery, M., Kimmig, H., and Fischer, B. The role of fixation and visual attention in the occurrence of express saccades in man. Eur. Arch. Psychiat. Neurol. Sci. 235: 269-275, 1986.

McIlwain, J. T. Point images in the visual system: new interest in an old idea. Trends Neurosci. 9: 354-358, 1986.

Munoz, D. P., Waitzman, D. M., And Wurtz, R. H. Activity of neurons in monkey superior colliculus during interrupted saccades. J. Neurophysiol. 75: 2562-2580, 1996.

MunOz, D. P. AND WuRTz, R. H. Role of the rostral superior colliculus in active visual fixation and execution of express saccades. J. Neurophysiol. 67: 1000-1002, 1992.

MunOz, D. P. AND Wurtz, R. H. Fixation cells in monkey superior colliculus. I. Characteristics of cell discharge. J. Neurophysiol. 70: 559-575, 1993a.

Munoz, D. P. And Wurtz, R. H. Fixation cells in monkey superior colliculus. II. Reversible activation and deactivation. J. Neurophysiol. 70: 576589, 1993 b.

MunOz, D. P. AND WurTz, R. H. Saccade-related activity in monkey superior colliculus. I. Characteristics of burst and buildup cells. J. Neurophysiol. 73: 2313-2333, 1995a.

MunOZ, D. P. AND Wurtz, R. H. Saccade-related activity in monkey superior colliculus. II. Spread of activity during saccades. J. Neurophysiol. 73: 2334-2348, 1995 b.

Paré, M. And GuitTon, D. The fixation area of the cat superior colliculus: effects of electrical stimulation and direct connection with brainstem omnipause neurons. Exp. Brain Res. 101: 109-122, 1994.

Paré, M. And Munoz, D. P. Saccadic reaction time in the monkey: ad- 
vanced preparation of oculomotor programs is primarily responsible for express saccade occurrence. J. Neurophysiol. 76: 3666-3681, 1996.

Pierrot-Deseilligny, C., Rivaud, S., Gaymard, B., and Agid, Y. Cortical control of reflexive visually-guided saccades. Brain 114: 1473-1485, 1991.

Rafal, R., Smith, J., Krantz, J., Cohen, A., and Brennan, C. Extrageniculate vision in hemianopic humans: saccade inhibition by signals in the blind field. Science Wash. DC. 250: 118-121, 1990.

Reuter-Lorenz, P. A., Hughes, H. C., And Fendrich, R. The reduction of saccadic latency by prior offset of the fixation point: an analysis of the gap effect. Percept. Psychophys. 49: 167-175, 1991.

Reuter-Lorenz, P. A., OonK, H. M., Barnes, L. L., And Hughes, H. C. Effects of warning signals and fixation point offsets on the latencies of pro versus anti-saccades: implications for an interpretation of the gap effect. Exp. Brain Res. 103: 287-293, 1995.

Rizzolatti, G., Camarda, R., Grupp, L. A., and Pisa, M. Inhibitory effects of remote visual stimuli on visual responses of cat superior colliculus: spatial and temporal factors. J. Neurophysiol. 37: 1262-1275, 1974.

Ross, L. E. AND Ross, S. M. Saccade latency and warning signals: stimulus onset, offset, and change as warning events. Percept. Psychophys. 27: 251-257, 1980.

Ross, S. M. AND Ross, L. E. Saccade latency and warning signals: effects of auditory and visual stimulus onset and offset. Percept. Psychophys. 29: 429-437, 1981.

SASLOW, M. G. Effects of components of displacement-step stimuli upon latency for saccadic eye movement. J. Opt. Soc. Am. 57: 1024-1029, 1967.

Schiller, P. H., True, S. D., AND Conway, J. L. Deficits in eye movements following frontal eye-field and superior colliculus ablations. J. Neurophysiol. 44: 1175-1189, 1980.

SPARKS, D. L. Translation of sensory signals into commands for control of saccadic eye movements: role of primate superior colliculus. Physiol. Rev. 66: 118-171, 1986.
Sparks, D. L. AND HaRTwich-YounG, R. The deep layers of the superior colliculus. In: The Neurobiology of Saccadic Eye Movements, edited by R. H. Wurtz and M. E. Goldberg. Amsterdam: Elsevier, 1989, p. $213-$ 255.

SPRAGUE, J. M. Interaction of cortex and superior colliculus in mediation of visually guided behavior in the cat. Science 153: 1544-1547, 1966.

WALKER, R. AND FINDLAY, J. M. Saccadic eye movement programming in unilateral neglect. Neuropsychologia 34: 493-508, 1996.

Walker, R., Findlay, J. M., Young, A. W., and Welch, J. Disentangling neglect and hemianopia. Neuropsychologia 29: 1019-1027, 1991.

WALKER, R., KENTRIDGE, R. W., AND FindLAY, J. M. Independent contributions of the orienting of attention, fixation offset and bilateral stimulation on human saccadic latency. Exp. Brain Res. 103: 294-310, 1995.

Wallace, S. F., Rosenquist, A. C., And Sprague, J. M. Recovery from cortical blindness mediated by destruction of non tectotectal fibres in the commisure of the superior colliculus in the cat. J. Comp. Neurol. 284: 429-450, 1989.

Wallace, S. F., Rosenquist, A. C., and Sprague, J. M. Ibotenic acid lesions of the lateral substantia nigra restores visual orientation behaviour in the hemianopic cat. J. Comp. Neurol. 296: 222-252, 1990.

Weber, H., Aiple, F., Fischer, B., And Latanov, A. Dead zone for express saccades. Exp. Brain Res. 89: 214-222, 1992.

WeBER, H. AND FISCHER, B. Differential effects of non-target stimuli on the occurrence of express saccades in man. Vision Res. 34: 1883-1891, 1994.

Wenban-Smith, M. G. And Findlay, J. M. Express saccades: is there a separate population in humans? Exp. Brain Res. 87: 218-222, 1991.

WurTZ, R. H., Richmond, B. J., AND JudGE, S. J. Vision during saccadic eye movements. III. Visual interactions in monkey superior colliculus J. Neurophysiol. 43: 1168-1180, 1980.

Wyman, D. and Steinman, R. M. Latency characteristics of small saccades. Vision Res. 13: 2173-2175, 1973. 